\title{
Improving the lifestyle and heart health of siblings and offspring of patients who had a heart attack before the age of 55 in men or the age of 65 in women
}

\author{
Condition category \\ Circulatory System \\ Date applied \\ 18/06/2020 \\ Date assigned \\ 06/08/2020 \\ Last edited \\ $13 / 12 / 2021$ \\ Prospective/Retrospective \\ Prospectively registered \\ Overall trial status \\ Ongoing \\ Recruitment status \\ Recruiting
}

\section{Plain English Summary}

\section{Background and study aims}

Smoking, poor diet, and lack of exercise are associated with obesity, poor blood pressure, glucose and cholesterol control, all being risk factors that lead to coronary heart disease. However, if lifestyle factors such as cigarette smoking, physical inactivity and unhealthy diet are modified, there might be a substantial decrease in coronary heart disease risk. If people are screened and identified, this will provide an opportunity for prevention to take place before the establishment of the disease, hence preventing or delaying the disease. The aim of this study is to evaluate the feasibility and acceptability of a preventive cardiology programme designed to promote risk reduction in a sample of relatives of patients with premature coronary heart disease.

Who can participate?

People aged 30 and older who have a brother or a sister or parents who had a heart attack before the age of 55 in men or the age of 65 in women

What does the study involve?

The study will consist of four separate visits of around 1 hour each (baseline assessment, CRISO lifestyle intervention programme/verbal lifestyle advice only, 6-month follow-up, 12-month follow-up). Participants are randomly allocated to the intervention group or the control group. Participants in the intervention group will have an initial face-to-face session (evaluation of modifiable risk factors, two-way risk factor communication, education and individualised counselling to set up a cardioprotective plan) followed by weekly telephone-based cardioprotective reinforcement sessions for up to 12 weeks. Participants in the control group will have a limited intervention consisting of one face-to-face session of lifestyle advice only.

What are the possible benefits and risks of participating?

Benefits could be weight, blood pressure and blood glucose improvement. The assessment techniques are safe and potential risks are very minimal. Some bruising is possible after blood sampling.

Where is the study run from?

Mater Dei Hospital (Malta)

When is the study starting and how long is it expected to run for?

June 2016 to September 2023 
Who is funding the study?

University of Malta (Malta)

Who is the main contact?

Justin Lee Mifsud

justin-lee.mifsud@um.edu.mt

\section{Trial website}

\section{Contact information}

\section{Type}

Scientific

\section{Primary contact}

Mr Justin Lee Mifsud

\section{ORCID ID}

https://orcid.org/oooo-0oo1-5380-9418 [https://orcid.org/oooo-0oo1-5380-9418]

\section{Contact details}

University of Malta

Office 38

Faculty of Health Sciences

Msida

MSD 2080

Malta

+356 (o)79433879

justin-lee.mifsud@um.edu.mt [mailto:justin-lee.mifsud@um.edu.mt]

\section{Type}

Scientific

\section{Additional contact}

Prof Joseph Galea

\section{ORCID ID}

[]

\section{Contact details}

University of Malta

Faculty of Medicine and Surgery

Msida

MSD 2080

Malta +356 (o) 99823852

joseph.f.galea@um.edu.mt [mailto:joseph.f.galea@um.edu.mt]

\section{Additional identifiers}




\section{EudraCT number}

Nil known

\section{IRAS number}

\section{ClinicalTrials.gov number}

Nil known

\section{Protocol/serial number}

HECo3 CTo1/2O

\section{Study information}

\section{Scientific title}

Feasibility and acceptability of a coronary risk reduction intervention for siblings and offspring of patients with premature coronary heart disease

\section{Acronym}

CRISO

\section{Study hypothesis}

The aim of this study is to evaluate the feasibility and acceptability of a drafted preventive cardiology programme, designed to promote risk reduction in a sample of asymptomatic first-degree relatives of patients with premature coronary heart disease (CHD).

\section{Ethics approval}

1. Approved 04/08/2020, University of Malta Research Ethics Committee (University of Malta, Msida MSD 2080; +356 (o)23402340; research-ethics.ms@um.edu.mt), ref: UNIQUE FORM ID: 3756_191119 2. Approved 04/08/2020, Health Ethics Committee Malta (Health Ethics Committee, Directorate for Health Information \& Research 95, Gwardamangia Hill, Pieta' - Malta PTA 1313 address; +356

(o)25599000; hec@gov.mt), ref: HECo3 CTo1/20

\section{Study design}

Randomised single-centre two-armed group randomized controlled trials with embedded interviews

\section{Primary study design}

Interventional

\section{Secondary study design}

Randomised controlled trial

\section{Trial setting}

Hospitals

\section{Trial type}

Prevention 


\title{
Patient information sheet
}

\author{
See additional files
}

\section{Condition}

First degree relatives of patients with premature coronary heart disease

\section{Intervention}

Block randomization will be applied to balance out sample size between intervention group and control group. Block randomization of four rows of relatives, using an opaque sealed envelope technique, will be implemented for this study.

Intervention: Participants will have an initial face-to-face session (evaluation of modifiable risk factors, twoway risk factor communication, education and individualised counselling to set up a cardio-protective plan) followed by weekly telephone-based cardio-protective reinforcement sessions for up to 12 weeks.

Control: Participants will have limited intervention, consisting of one face to face session of lifestyle advice only.

\section{Intervention type}

Behavioural

\section{Phase}

\section{Drug names}

\section{Primary outcome measure}

Feasibility measured using records of the number of:

1. Relatives recruited via patients - measured at recruitment stage

2. Relatives recruited via social media - measured at recruitment stage

3. Relatives recruited via poster method (local councils, pharmacies, social groups) - measured at recruitment stage

4. Relatives who expressed interest in the study - measured at recruitment stage

5. Relatives who are potentially eligible, with reasons for ineligibility - measured at recruitment stage

6. Relatives who agreed to participate in the trial - measured at recruitment stage

7. Relatives lost to follow-up (retention and drop-out rate) - measured at 12 months

8. Sessions delivered successfully - measured at 12 months

Furthermore, the feasibility of the intervention will include the time taken to:

1. Complete cardiovascular risk assessment - measured at baseline, 6 months and 12 months.

2. Deliver the intervention (in-person session and telephone sessions) - time recorded for the individualized intervention session (in-person session and telephone sessions)

Acceptability will be evaluated by determining how well the intervention is received by first degree relatives and the extent to which this intervention could meet the needs of first-degree relatives. This requires data of the participants' most valued intervention components in terms of being supportive and the participants' views of the quality of interactions during intervention sessions, along with how well the intervention targets can be incorporated into participants' personal lifestyles, taking into consideration any difficulties that were experienced while attempting to comply with the programme goals. To collect this data, post-intervention questionnaires and interviews will be used. This data will determine which part of the intervention the participants found most supportive and which parts may require further refinement. Evaluated at 12 months.

\section{Secondary outcome measures}

1. Smoking status measured using a CO monitor at baseline, 6 months and 12 months.

2. Dietary status measured using the Mediterranean diet score at baseline, 6 months and 12 months

3. Physical activity status measured using the RAPA score at baseline, 6 months and 12 months 
4. Blood biochemicals (lipids, HbA1c) measured using the Roche COBAS analyser, at baseline, 6 months and 12 months

5. Resting physiological measurements measured using an Omron blood pressure monitor, measured two to three times by auscultation, and an average will be taken as the final measurement, and heart rate measured manually by radial pulse at baseline, 6 months and 12 months

6. Anthropometric measures (BMI, waist circumference) measured at baseline, 6 months and 12 months

7. Heart age score measured using the Heart Age calculator at baseline, 6 months and 12 months

8. Health literacy measured using the Health Literacy Score at baseline, 6 months and 12 months

\section{Overall trial start date}

01/o6/2016

\section{Overall trial end date}

$01 / 09 / 2023$

\section{Reason abandoned (if study stopped)}

\section{Eligibility}

\section{Participant inclusion criteria}

1. First-degree relatives of patients with premature CHD

2. Aged 30 years and older

3. Males and females

4. Maltese ethnicity

5. Seeking cardiovascular risk assessment

6. No history of cardiovascular disease

\section{Participant type}

Healthy volunteer

\section{Age group}

Adult

\section{Gender}

Both

\section{Target number of participants}

100

\section{Participant exclusion criteria}

1. Participants must not have a history of rheumatoid arthritis and chronic kidney disease

2. Participants must not have contraindications against physical activities and are able to go up a flight of stairs comfortably

3. Participants must not be pregnant

\section{Recruitment start date}

$01 / 09 / 2020$

\section{Recruitment end date}

01/09/2022 


\section{Locations}

\section{Countries of recruitment}

Malta

\section{Trial participating centre}

\section{Mater Dei Hospital}

Cardiac rehabilitation services

Msida

MSD 2090

Malta

\section{Sponsor information}

\section{Organisation}

University of Malta

\section{Sponsor details}

Faculty of Medicine and Surgery

Block A

Level o

Mater Dei Hospital

Msida

MSD2090

Malta

+356 (o)23401138

umms@um.edu.mt [mailto:umms@um.edu.mt]

\section{Sponsor type}

University/education

\section{Website}

http://www.um.edu.mt/ [http://www.um.edu.mt/]

\section{GRID}

grid.4462.4 [https://www.grid.ac/institutes/grid.4462.4]

\section{Funders}

\section{Funder type}

University/education

\section{Funder name}

University of Malta

\section{Alternative name(s)}

\section{Funding Body Type}




\section{Funding Body Subtype}

\section{Location}

\section{Results and Publications}

\section{Publication and dissemination plan}

Planned publication in a high-impact peer-reviewed journal. The researchers are hoping to publish their study protocol and data analysis plan.

2020 video in https://www.um.edu.mt/newspoint/notices/opportunities/2020/o8/phd-studies-justinmifsud [https://www.um.edu.mt/newspoint/notices/opportunities/2020/o8/phd-studies-justin-mifsud] (added 28/09/2020)

\section{Intention to publish date}

$31 / 12 / 2025$

\section{Individual participant data (IPD) sharing statement}

Data is being handled, coded and stored in accordance with the Data Protection Act 2018, in line with GDPR EU 2016/679. Raw data will be stored in an encrypted personal computer and only the principal researcher (Justin L. Mifsud) and the supervisor (Joseph Galea) will have access to such data. When the data is analysed, it is planned to be published in a peer-reviewed journal and reported to reflect on all the predefined aims and objectives published in the study protocol.

(added 13/12/2021)

Availability of data and materials

The anonymised participant datasets generated and/or analysed during the current study will be made available in the University of Malta institutional open access data repository

https://www.um.edu.mt/library/oar [https://www.um.edu.mt/library/oar] /.

\section{Participant level data}

Stored in publicly available repository

\section{Trial outputs}

\begin{tabular}{|c|c|c|c|}
\hline $\mathbf{u}$ & Details & $\begin{array}{c}\text { Date } \\
\text { created }\end{array}$ & $\begin{array}{c}\text { Date } \\
\text { added }\end{array}$ \\
\hline
\end{tabular}

Participant information sheet

[/editorial/retrieveFile/861df5d4-

3fd1-407a-a8d628/09/2020 No Yes

9ded7ddb7fe5/38441]

Protocol article

[https://doi.org/10.1186/s40814-

021-00874-4]

\section{Additional files}

- ISRCTN21559170_PIS.pdf [/editorial/retrieveFile/861df5d4-3fd1-407a-a8d6-9ded7ddb7fe5/38441] Uploaded 28/09/2020

\section{Editorial Notes}

13/12/2021: The following changes were made to the trial record: 1 . Publication reference added. 2. The data sharing statement has been updated. 28/09/2020: The participant information sheet has been uploaded. 
01/09/2020: Internal review. 03/08/2020: Trial's existence confirmed by Health Ethics Committee Malta. 